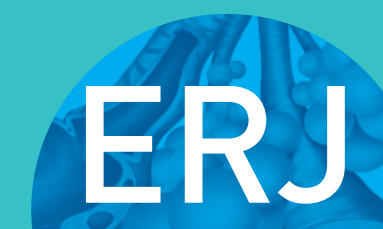

open research

\title{
Upper airway involvement in bronchiectasis is marked by early onset and allergic features
}

\author{
Michal Shteinberg ${ }^{1,2,3}$, Najwan Nassrallah ${ }^{4}$, Jenny Jrbashyan ${ }^{5}$, Nechama Uri ${ }^{5}$, \\ Nili Stein ${ }^{6}$ and Yochai Adir ${ }^{1,3}$
}

Affiliations: ${ }^{1}$ Pulmonology Institute, Carmel Medical Center, Haifa, Israel. ${ }^{2}$ Cystic Fibrosis Center, Carmel Medical Center, Haifa, Israel. ${ }^{3} \mathrm{~B}$. Rappaport Faculty of Medicine, Technion - Israel Institute of Technology, Haifa, Israel. ${ }^{4}$ Dept of Radiology, Carmel Medical Center, Haifa, Israel. ${ }^{5}$ Dept of Otolaryngology, Carmel Medical Center, Haifa, Israel. ${ }^{6}$ Dept of Community Medicine and Epidemiology, Carmel Medical Center, Haifa, Israel.

Correspondence: Michal Shteinberg, Pulmonary Institute, Carmel Medical Center, 7 Michal Street, Haifa 3463209, Israel. E-mail: michalsh@technion.ac.il

ABSTRACT The association of bronchiectasis with chronic rhinosinusitis (CRS) has been reported. However, apart from primary ciliary dyskinesia (PCD) and cystic fibrosis (CF), predisposing conditions have not been established. We aimed to define clinical and laboratory features that differentiate patients with bronchiectasis with upper airway symptoms (UASs) and without PCD from patients without UASs.

We reviewed charts of adults with bronchiectasis, excluding CF and PCD. UASs were defined as nasal discharge most days of the year, sinusitis or nasal polyps. Laboratory data included IgG, total IgE, blood eosinophils, sputum bacteriology and lung function. A radiologist blinded to UAS presence scored bronchiectasis (Reiff score) and sino-nasal pathology (Lund-Mackay score).

Of 197 patients, for the 70 (35\%) with UASs, symptoms started earlier (34 \pm 25 versus $46 \pm 24$ years; $\mathrm{p}=0.001$ ), disease duration was longer (median 24 versus 12 years; $\mathrm{p}=0.027$ ), exacerbations were more frequent (median 3 versus 2 per year; $\mathrm{p}=0.14$ ), and peripheral blood eosinophil (median 230 versus $200 \mu \mathrm{L}^{-1} ; \mathrm{p}=0.015$ ) and total IgE (median 100 versus $42 \mathrm{IU} \cdot \mathrm{mL}^{-1} ; \mathrm{p}=0.085$ ) levels were higher. The sinus computed tomography score was independently associated with exacerbations, with 1 point on the Lund-Mackay score associated with a 1.03 -fold increase in the number of exacerbations per year $(95 \% \mathrm{CI}$ $1.0-1.05 ; \mathrm{p}=0.004)$.

These findings may implicate a higher disease burden in patients with UASs. We hypothesise that UASs precede and may in some cases lead to the development of bronchiectasis.

@ERSpublications

Involvement of the upper airway in patients with bronchiectasis is associated with an early age of onset and allergic features http://ow.ly/1BuK30gWDrN

Cite this article as: Shteinberg M, Nassrallah N, Jrbashyan J, et al. Upper airway involvement in bronchiectasis is marked by early onset and allergic features. ERJ Open Res 2018; 4: 00115-2017 [https://doi.org/10.1183/23120541.00115-2017].

Received: Sept 202017 | Accepted after revision: Nov 052017

Conflict of interest: None declared.

Copyright $\odot$ ERS 2018. This article is open access and distributed under the terms of the Creative Commons Attribution Non-Commercial Licence 4.0. 


\section{Introduction}

Several clinical entities that affect the lung airways involve the upper airway. Examples are the association of asthma and chronic rhinosinusitis (CRS) in patients with atopy [1-3], and the involvement of the upper and lower airways in patients with cystic fibrosis (CF) and primary ciliary dyskinesia (PCD) [4]. In these entities, a common mechanism affects the upper and lower airways. In bronchiectasis, the involvement of the upper airway is well established [5-8]. CRS was reported in 34-45\% of patients with bronchiectasis in studies from China and Japan [7, 9], and in 75\% of European patients with bronchiectasis [8, 10]. Patients with CRS and bronchiectasis have also been found to experience more exacerbations than those without CRS $[6-8,11]$. However, comparisons of lung function and bacterial colonisation between patients with and without upper airway involvement have shown contradictory findings [6-8].

The mechanisms of upper airway involvement in bronchiectasis other than CF and PCD have not been well established. CRS was found to be more prevalent in idiopathic than in post-infectious bronchiectasis in one study [12], suggesting that "idiopathic" but not "post-infectious" bronchiectasis results from diffuse inflammation that involves the upper and lower airways. An allergic tendency causing CRS and inflammation of the lower airway has also been suggested [1, 13], although to the best of our knowledge this has not been established in bronchiectasis, excluding bronchiectasis associated with allergic bronchopulmonary aspergillosis (ABPA). Although the association of CRS with bronchiectasis is clear, the pathogenesis of this association is unknown: is CRS causing bronchiectasis, is bronchiectasis causing CRS or is a common mechanism predisposing to both?

The aim of this study was to explore differences between patients with bronchiectasis, with and without upper airway involvement. We hypothesised that allergic features are more prevalent among bronchiectasis patients with than without upper airway involvement.

\section{Methods}

Patients and setting

Our study was conducted in an adult bronchiectasis referral clinic and the protocol was approved by the Helsinki committee at Carmel Medical Center, Haifa, Israel (CMC-60-14). Patients were evaluated for symptoms of CRS and patients' records were reviewed for sino-nasal disease. Aetiological workup done for all patients with bronchiectasis included a detailed history (age of onset, presence of sinusitis, rhinitis or nasal polyps, history of pneumonia, infertility or ectopic pregnancies, consanguinity or a family history of upper or lower airway disease, middle ear infections, or situs abnormalities). All patients had spirometry with reversibility testing, sputum microbiological testing, and were tested for immunoglobulins and total IgE. For a diagnosis of asthma, patients had to either have a documented obstructive spirometry with reversibility or a positive methacholine challenge. Patients with features suggestive of CF were referred for sweat testing, followed by additional tests if necessary [14]. Patients with features suggestive of PCD were screened with nasal nitric oxide and additional tests if low [4].

Exacerbations were defined as episodes of worsening cough or dyspnoea that was treated with a course of antibiotics, as documented from the patients' electronic medical records and pharmacy records.

\section{Radiological evaluation}

Sinus and chest computed tomography (CT) scans were analysed by a radiologist (N.N.) blinded to the presence of upper airway symptoms (UASs). Chest CT scans were scored for bronchiectasis severity by the modified Reiff score [15]. Sinus involvement was scored using the Lund-Mackay system, with scores ranging from 0 to 24 [16].

\section{Definition of upper airway involvement}

CRS was diagnosed based on the criteria of the European Position Paper on Rhinosinusitis and Nasal Polyps (EP ${ }^{3} \mathrm{OS}$ ) [17], which require persistent symptoms, as well as CT findings typical of rhinosinusitis. A Lund-Mackay score $\geqslant 4$ was considered as positive for CRS, as previously suggested [18]. PCD was diagnosed according to clinical and laboratory criteria [4]. However, patients with a strong clinical suspicion of PCD (e.g. presence of dextrocardia), but not meeting laboratory criteria, were also excluded from the study. Age at onset of symptoms was determined from patient history. The date of bronchiectasis diagnosis was the date of the first CT scan detecting bronchiectasis.

Three analyses were done to differentiate between patients with and without upper airway involvement. 1) We differentiated patients with and without UASs based on reported symptoms only, regardless of the availability or findings in sinus CT. 2) We excluded patients with a discrepancy between UASs and sinus CT findings, using a Lund-Mackay score of 4 as the cut-off for upper airway involvement. Analyses with a Lund-Mackay cut-off of 6 and 10 were also performed. Patients without sinus CT scans were included in the second analysis according to the presence or absence of UASs. 3) We included only patients with sinus 
CT scans that were available for review. In this analysis, patients meeting both clinical and radiological criteria for the presence or absence of CRS were included. This analysis refers to patients for whom CRS was present or absent.

\section{Laboratory and clinical findings}

Immunoglobulins were taken during the initial assessment at the bronchiectasis clinic. Eosinophil counts were taken as the median of peripheral blood eosinophil counts during the past 3 years. Sputum cultures were considered positive for a pathogen if at least one culture was positive. Forced expiratory volume in $1 \mathrm{~s}$ $\left(\mathrm{FEV}_{1}\right)$ was taken as the best value during the previous year and presented as the percentage of predicted.

\section{Statistical analysis}

Statistical analysis was performed using SPSS Statistics version 22 (IBM, Armonk, NY, USA). Continuous variables were compared between the groups using the Mann-Whitney test for nonnormal distribution. Categorical variables were compared using the Chi-squared test. $\mathrm{p}<0.05$ was considered statistically significant. Correlation of the number of exacerbations with clinical and demographic characteristics was examined using the Pearson or Spearman correlation, as appropriate.

\section{Results}

214 adults with non-CF bronchiectasis from March 2014 to December 2015 were included. 12 patients met criteria for PCD and two others had clinical features strongly suggestive of PCD, but did not meet laboratory criteria. These 14 patients were given a diagnosis of probable PCD and were excluded from the study. Three other patients had incomplete clinical and laboratory data, and were also excluded. Therefore, 197 patients were included in the study. 70 patients (35\%) had UASs and 127 (65\%) did not have UASs. For 11 patients with UASs, sinus CT Lund-Mackay scores were $\leqslant 3$. Similarly, for 23 patients without UASs, sinus CT Lund-Mackay scores were $\geqslant 4$. These patients with a discrepancy between clinical and radiological findings were excluded from the second analysis. Finally, for 34 out of 70 with UASs and for 38 out of 127 without UASs, a sinus CT scan compatible with the presence and absence of CRS, respectively, was available for review, and they were included in the third analysis (figure 1).

Although the age at referral to the bronchiectasis clinic did not differ between the two groups, patients with UASs had a significantly younger median age of symptom onset ( $34 \pm 25$ versus $46 \pm 24$ years; $\mathrm{p}=0.001$ ). Compared with patients without UASs, among those with UASs, post-infectious aetiology was significantly less common (12.9\% versus $29.1 \%$; $\mathrm{p}=0.01)$, the prevalence of asthma was higher $(14.3 \%$

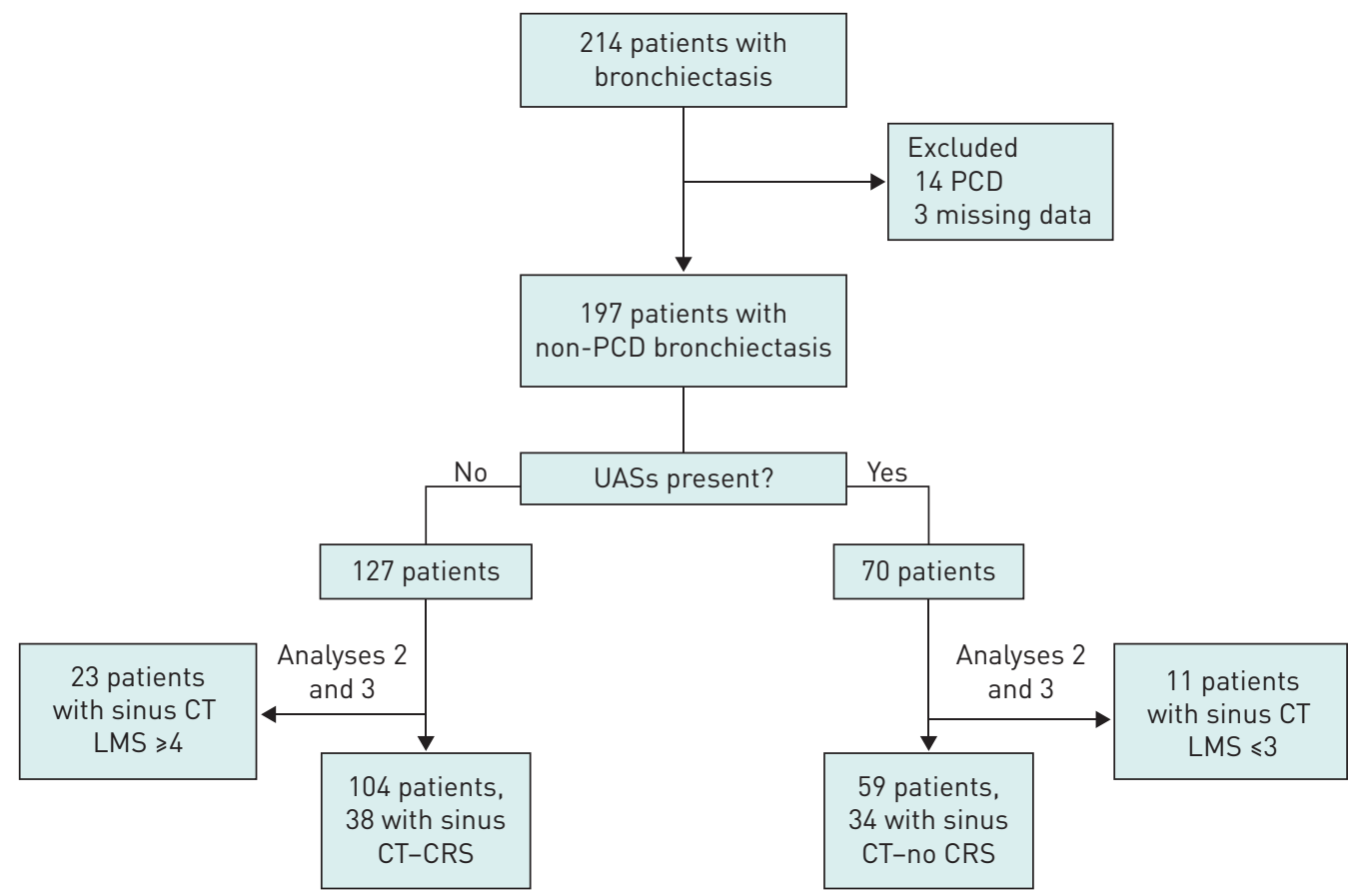

FIGURE 1 Flowchart of patients with bronchiectasis included in the analyses. PCD: primary ciliary dyskinesia; UAS: upper airway symptom; CT: computed tomography; LMS: Lund-Mackay score; CRS: chronic rhinosinusitis. 
versus $5.5 \% ; \mathrm{p}=0.036)$, the median peripheral eosinophil count was higher $\left(230\right.$ versus $\left.200 \mu \mathrm{L}^{-1} ; \mathrm{p}=0.015\right)$ and the total IgE level was higher (median 100 versus $42 \mathrm{IU} \cdot \mathrm{mL}^{-1} ; \mathrm{p}=0.085$ ), but did not reach statistical significance. Patients with UASs had more exacerbations (median 3 versus 2; $\mathrm{p}=0.05$ ) despite a nonsignificant difference in median $\mathrm{FEV}_{1}(86 \%$ versus $75 \%$; $\mathrm{p}=0.134)$ and a lower CT Reiff score $(6$ versus $7 ; \mathrm{p}=0.031)$ (table 1).

Due to the retrospective study design, we were concerned that classifying patients only on the basis of symptoms would either over- or underestimate upper airway involvement. To differentiate more precisely, without excluding the many patients without available sinus CT, we repeated the analysis after excluding patients with a discrepancy between symptoms and CT findings (Lund-Mackay score $\leqslant 3$ with UASs or $\geqslant 4$ without UASs). The results were similar except for the CT severity score (Reiff score) of bronchiectasis, which no longer showed a statistically significant difference (table 2).

In a third analysis, which included only patients with sinus CT available for review, CRS was defined as the presence of both UASs and a Lund-Mackay score $\geqslant 4$. The absence of CRS was defined as both a lack of UASs and a Lund-Mackay score $\leqslant 3$. This analysis included 38 patients without CRS and 34 with CRS; the time from symptom onset to referral to the bronchiectasis service remained statistically significant. Total eosinophil count remained significantly higher in patients with than without CRS, with the difference more pronounced than in the former analyses (median eosinophil count 275 versus $175 \mu \mathrm{L}^{-1}$; $\mathrm{p}=0.015$ ). Other variables showed trends toward the same differences between patients with and without CRS as were found in the previous analyses, but without statistical significance (table 3).

TABLE 1 Characteristics of bronchiectasis patients with and without upper airway symptoms (UASS)

\begin{tabular}{|c|c|c|c|}
\hline & No UASs & UASs & p-value \\
\hline Subjects & 127 & 70 & \\
\hline Female & $76(61.4)$ & $49(70.0)$ & 0.238 \\
\hline Age at onset years & $45.85 \pm 23.7$ & $33.65 \pm 24.7$ & 0.001 \\
\hline Current age years & $65.8 \pm 14.7$ & $60.2 \pm 16.7$ & 0.016 \\
\hline Time from onset to diagnosis years & $12.6(3.7-27.5)$ & $23.8(7.4-45.9)$ & 0.027 \\
\hline \multicolumn{4}{|l|}{ Aetiology" } \\
\hline Presence of asthma & $7(5.5)$ & 10 (14.3) & 0.036 \\
\hline Idiopathic aetiology & $56(44.1)$ & $34(48.6)$ & 0.546 \\
\hline COPD & $10(7.9)$ & 2 (2.9) & 0.219 \\
\hline Post-infectious aetiology & $37(29.1)$ & $9(12.9)$ & 0.010 \\
\hline Gastro-oesophageal reflux & 0 & $1(1.4)$ & NA \\
\hline Radiation therapy & $6(4.7)$ & $2(2.9)$ & NA \\
\hline Inflammatory bowel disease & 0 & $1(1.4)$ & NA \\
\hline Immune deficiency & 5 (3.9) & $5(7.1)$ & NA \\
\hline Rheumatoid arthritis & $3(2.4)$ & $1(1.4)$ & NA \\
\hline Others/not completed evaluation & $3(2.4)$ & 6 (8.5) & NA \\
\hline \multirow[t]{2}{*}{ Eosinophils $\mu \mathrm{L}^{-1}$} & $n=123$ & $\mathrm{n}=67$ & 0.015 \\
\hline & $200(100-280)$ & $230(100-400)$ & \\
\hline \multirow[t]{2}{*}{$\lg \mathrm{E} I U \cdot \mathrm{mL}^{-1}$} & $\mathrm{n}=75$ & $n=50$ & 0.085 \\
\hline & $42(11-119)$ & $100(11.8-328)$ & \\
\hline \multirow[t]{2}{*}{$\lg G \operatorname{IU} \cdot \mathrm{mL}^{-1}$} & $\mathrm{n}=98$ & $\mathrm{n}=62$ & 0.944 \\
\hline & 1160 (1005-1388) & 1182 (945.3-1420) & \\
\hline \multirow[t]{2}{*}{ FEV $1 \%$ pred } & $n=118$ & $\mathrm{n}=66$ & 0.134 \\
\hline & $77.5 \pm 26.0$ & $83.5 \pm 25.6$ & \\
\hline \multirow[t]{2}{*}{ Chest CT Reiff score } & $n=109$ & $n=56$ & 0.031 \\
\hline & $7(6-12)$ & $6(4-8.75)$ & \\
\hline \multirow[t]{2}{*}{ Sinus CT Lund-Mackay score } & $n=61$ & $n=45$ & $<0.0001$ \\
\hline & $2(0-6)$ & $9(3.5-12.5)$ & \\
\hline \multirow[t]{2}{*}{ Exacerbations per year } & $\mathrm{n}=122$ & $n=68$ & 0.135 \\
\hline & $2(1-4)$ & $3(2-4)$ & \\
\hline \multirow[t]{2}{*}{ Occurrence of exacerbation in previous year } & $n=122$ & $n=68$ & 0.134 \\
\hline & $104(85.2)$ & $63(92.6)$ & \\
\hline
\end{tabular}

Data are presented as $\mathrm{n}, \mathrm{n}(\%)$, mean $\pm \mathrm{SD}$ or median (interquartile range), unless otherwise stated. COPD: chronic obstructive pulmonary disease; FEV1: forced expiratory volume in $1 \mathrm{~s}$; $\mathrm{CT}$ : computed tomography; NA: not available. ${ }^{\#}$ : more than one aetiology may be present for some patients and therefore percentages exceed $100 \%$. $p<0.05$ was considered significant. 
TABLE 2 Characteristics of patients with bronchiectasis, with and without upper airway

symptoms (UASs), excluding patients with either UASs and a Lund-Mackay score $\leqslant 3$ or lack of UASs and Lund-Mackay score $\geqslant 4$

\begin{tabular}{|c|c|c|c|}
\hline & No UASs & UASs & p-value \\
\hline Subjects & 104 & 59 & \\
\hline Female & 64 (61.5) & 40 (67.8) & 0.424 \\
\hline Age at onset years & $45.01 \pm 23.5$ & $33.5 \pm 23.9$ & 0.007 \\
\hline Age at diagnosis years & $64.0 \pm 15.0$ & $59.4 \pm 17.7$ & 0.201 \\
\hline Time from onset to diagnosis years & $13.0(3.6-27)$ & $24(7.7-40.6)$ & 0.020 \\
\hline Presence of asthma & $5(4.8)$ & 7 (11.9) & 0.122 \\
\hline Idiopathic aetiology & $50(48.1)$ & $31(52.5)$ & 0.584 \\
\hline Post-infectious aetiology & $31(29.8)$ & 7 (11.9) & 0.009 \\
\hline Eosinophils $\mu \mathrm{L}^{-1}$ & $200(50-500)$ & $290(158-488)$ & 0.002 \\
\hline $\operatorname{IgE} I U \cdot \mathrm{mL}^{-1}$ & $\begin{array}{c}n=61 \\
42(11-114)\end{array}$ & $\begin{array}{c}n=42 \\
100(13-305)\end{array}$ & 0.066 \\
\hline $\operatorname{IgG} I U \cdot \mathrm{mL}^{-1}$ & $\begin{array}{c}n=79 \\
1169(1007-1350)\end{array}$ & $\begin{array}{c}n=53 \\
1184(962-1449)\end{array}$ & 0.747 \\
\hline FEV $1 \%$ pred & $81 \pm 26$ & $81 \pm 26$ & 0.961 \\
\hline Chest CT Reiff score & $\begin{array}{c}n=86 \\
7(5-12)\end{array}$ & $\begin{array}{c}n=46 \\
6(5-10)\end{array}$ & 0.632 \\
\hline Sinus CT Lund-Mackay score & $\begin{array}{c}n=38 \\
0.5(0-2)\end{array}$ & $\begin{array}{c}n=34 \\
12(8-13.0)\end{array}$ & $<0.0001$ \\
\hline Exacerbations per year $\mathbf{n}$ & $2(1-4)$ & $3(2-4)$ & 0.035 \\
\hline Occurrence of exacerbation in previous year & $\begin{array}{c}n=100 \\
86(86.0)\end{array}$ & $\begin{array}{c}n=58 \\
56(96.6)\end{array}$ & 0.034 \\
\hline
\end{tabular}

Data are presented as $\mathrm{n}, \mathrm{n}(\%)$, mean \pm SD or median (interquartile range), unless otherwise stated. FEV1: forced expiratory volume in $1 \mathrm{~s}$; CT: computed tomography. $\mathrm{p}<0.05$ was considered significant.

TABLE 3 Characteristics of patients with bronchiectasis, with and without chronic rhinosinusitis (CRS)

\begin{tabular}{|c|c|c|c|}
\hline & No CRS & CRS & p-value \\
\hline Subjects & 38 & 34 & 0.914 \\
\hline Female & $23(60.5)$ & $21(61.8)$ & 0.914 \\
\hline Age at onset years & $48.7 \pm 21.5$ & $39.5 \pm 23.9$ & 0.134 \\
\hline Age at diagnosis years & $65.6 \pm 13.4$ & $65.4 \pm 15.2$ & 0.644 \\
\hline Time from onset to diagnosis years & $11.8(2.6-26)$ & $27(4.3-41.8)$ & 0.046 \\
\hline Presence of asthma & $3(7.9)$ & $4(11.8)$ & 0.700 \\
\hline Idiopathic aetiology & 15 (39.5) & $18(52.9)$ & 0.252 \\
\hline Post-infectious aetiology & $10(26.3)$ & $4(11.8)$ & 0.119 \\
\hline Eosinophils $\mu \mathrm{L}^{-1}$ & $170(100-200)$ & $275(195-413)$ & 0.001 \\
\hline $\lg \mathrm{IU} \cdot \mathrm{mL}^{-1}$ & $\begin{array}{c}n=22 \\
60(21-267)\end{array}$ & $\begin{array}{c}n=21 \\
128(12-325)\end{array}$ & 0.610 \\
\hline $\operatorname{IgG} \mathrm{IU} \cdot \mathrm{mL}^{-1}$ & $\begin{array}{c}n=29 \\
1097(1000-1274)\end{array}$ & $\begin{array}{c}n=31 \\
1100(900-1377)\end{array}$ & 0.871 \\
\hline FEV $1 \%$ pred & $76.8 \pm 25.7$ & $81.6 \pm 24.9$ & 0.447 \\
\hline Chest CT Reiff score & $\begin{array}{c}n=35 \\
7(6-13)\end{array}$ & $\begin{array}{c}n=31 \\
6(5-8)\end{array}$ & 0.402 \\
\hline Sinus CT Lund-Mackay score & $0.5(0-2)$ & $12(7.8-13.0)$ & $<0.0001$ \\
\hline Exacerbations per year $\mathbf{n}$ & $3(1-4)$ & $4(2-5)$ & 0.064 \\
\hline Occurrence of exacerbation in previous year & $34(94.4)$ & $34(100)$ & 0.493 \\
\hline \multicolumn{4}{|c|}{$\begin{array}{l}\text { Data are presented as } n, n(\%), \text { mean } \pm \text { SD or median (interquartile range), unless otherwise stated. FEV1: } \\
\text { forced expiratory volume in } 1 \mathrm{~s} ; \mathrm{CT} \text { : computed tomography. }{ }^{\#} \text { : defined as UASs with evidence of sino-nasal } \\
\text { involvement on sinus CT (Lund-Mackay score } \geqslant 4 \text { ); }{ }^{\text {: }} \text { : patients without UASs and with a Lund-Mackay score } \\
\leqslant 3 . p<0.05 \text { was considered significant. }\end{array}$} \\
\hline
\end{tabular}


TABLE 4 Prevalence of bacteria in sputum or lavage cultures of patients with bronchiectasis

\begin{tabular}{lccccccc} 
& No UASs & UASs $^{\#}$ & $\mathbf{p}^{-v a l u e}$ & No CRS & CRS $^{+}$ & p-value $^{\text {1 }}$ \\
\hline Patients with sputum cultures available & 112 & 66 & & 31 & 32 & \\
Staphylococcus aureus & $7(6.3)$ & $8(12.1)$ & 0.173 & $0(0)$ & $4(12.5)$ & 0.113 \\
Haemophilus influenzae & $24(21)$ & $20(45.5)$ & 0.185 & $7(22.6)$ & $10(31.3)$ & 0.438 \\
Streptococcus pneumoniae & $6(5.4)$ & $5(7.6)$ & 0.539 & $1(3.2)$ & $2(6.3)$ & $>0.99$ \\
Pseudomonas aeruginosa & $39(34.8)$ & $18(27.3)$ & 0.297 & $14(45.2)$ & $9(28.1)$ & 0.160 \\
Nontuberculous mycobacteria & $12(11.3)$ & $5(7.6)$ & 0.424 & $2(6.7)$ & $3(9.1)$ & $>0.99$ \\
\hline
\end{tabular}

Data are presented as $\mathrm{n}$ or $\mathrm{n}(\%)$, unless otherwise stated. UAS: upper airway symptom; CRS: chronic rhinosinusitis. Patients with at least one positive culture were included. ${ }^{\#}$ : presence of persistent UASs or evidence of rhinitis or sinusitis on physical examination; " : patients without UASs and with a Lund-Mackay score $\leqslant 3 ;^{+}$: defined as UASs with evidence of sino-nasal involvement on sinus computed tomography (Lund-Mackay score $\geqslant 4$ ).

Patients with UASs had higher rates of positive growth of Staphylococcus aureus and Haemophilus influenzae, and less Pseudomonas aeruginosa in airway secretions than patients without UASs, although differences did not reach statistical significance (table 4).

In addition to the analysis based on a Lund-Mackay score cut-off of 4, we repeated the analyses using higher Lund-Mackay scores $(\leqslant 5$ versus $\geqslant 6$ and $\leqslant 9$ versus $\geqslant 10)$ to distinguish the presence or absence of CRS; similar differences were found between patients with the presence or absence of CRS. In this analysis, there was significantly less growth of P. aeruginosa among patients with CRS than without CRS when a Lund-Mackay score of 10 was taken as the cut-off (22.7\% versus $51 \%$; $=0.036)$.

A correlation between the number of exacerbations and clinical and laboratory parameters was examined using the Pearson or Spearman correlation, as appropriate. The Lund-Mackay score was the only parameter found to be significantly associated with the number of exacerbations, with a coefficient of 0.2 $(p=0.045)$. We analysed the correlation between the sinus CT Lund-Mackay score and exacerbations in the previous year. Patients with any abnormality on the sinus CT scan (Lund-Mackay score $\geqslant 1$ ) had a median (interquartile range) of $3(0-12)$ exacerbations per year, while patients with no abnormality on the sinus CT scan had $2(0-12)$ exacerbations per year $(\mathrm{p}=0.033)$. Similar differences were also found when the Lund-Mackay score cut-off was set at 6 and 10 points. We next performed a Poisson regression analysis between the Lund-Mackay score and the number of exacerbations. Each point on the Lund-Mackay score was associated with a 1.03 -fold increase in the number of exacerbations per year $(95 \%$ CI $1.0-1.05 ; \mathrm{p}=0.004)$.

\section{Discussion}

Our results indicate that in patients with upper airway involvement, symptoms start at an earlier age and that these patients had more allergic features, i.e. eosinophilia and elevated IgE. Asthma was more prevalent in patients with than without UASs $(14.3 \%$ versus $5.5 \% ; \mathrm{p}=0.036)$, but not very common in either group. Despite the lack of significant differences in the severity of lung function and radiological bronchiectasis, patients with UASs had significantly more frequent exacerbations, although no differences were found in the rate of hospitalisations.

In the current cohort, the rate of UASs among patients with bronchiectasis (35\%) was significantly lower than reported among European patients $(75-80 \%)[8,11,12,19]$, but similar to a more recent Chinese cohort [7] and to an earlier Japanese study [9]. Both our cohort and previous studies [6, 7, 20] found a significantly higher prevalence of CRS among patients with bronchiectasis than the $2-5.5 \%$ prevalence reported in the general population [21, 22]. This may suggest either that CRS predisposes to the development of bronchiectasis or that a common mechanism, such as allergy, is responsible for the two clinical entities.

Our evaluation included routine immunoglobulins, including IgE for most patients. When allergic features, such as elevated IgE or eosinophils, were noted, patients were referred to skin testing and Aspergillus-specific IgE to detect ABPA. The low prevalence of ABPA in our cohort compared with previous published series [23-25] is notable and may reflect a lower prevalence in our geographical area.

Our cohort consisted of Israeli patients of heterogeneous ethnic and genetic backgrounds: Ashkenazi and Eastern Jews, Muslim and Christian Arabs, and Druze. The genetic composition of our population may affect the prevalence of allergic diathesis, and explain, in part, differences in UASs and CRS prevalence 
between our cohort and others. Another possible explanation for the differences in prevalence is our exclusion of patients with features consistent with PCD (14 out of 220 patients initially screened). Upper airway involvement is almost universal among patients with PCD $[4,26]$; thus, if patients with PCD were included in our cohort, the expected rate of UASs would be $40 \%$ (84 out of 211). Another explanation for differences between our study and others is that the retrospective design may have resulted in underreporting by patients of their UASs. Indeed, in our cohort, 23 out of 127 patients who did not have symptoms of upper airway involvement had a sinus CT that showed sino-nasal pathology (Lund-Mackay score $\geqslant 4$ ). This number decreased to six patients when a Lund-Mackay score of 10 was used as the cut-off for upper airway involvement.

In all three analyses described herein, the reported age at onset of bronchiectasis symptoms (mainly productive cough) was significantly younger among patients with UASs. As the UAS and non-UAS groups were of similar age, the UAS group had a longer duration of illness. Despite this finding, patients with UASs did not have worse lung function or greater radiological severity than those without UASs. However, patients with UASs had significantly more frequent exacerbations (median 3 versus 2) than did those without UASs, similar to previous reports $[7,11]$. The contradiction between better lung function and radiological score, yet more frequent exacerbations among patients with UASs, may result from "upper airway exacerbations" driven by sino-nasal discharge and post-nasal drip. CRS is prevalent among children and adults; the mean age of onset was found to be $\sim 39$ years of age $[21,22]$. This is comparable to the age of symptom onset in our patients with UASs (median 34 years) and considerably lower than for those without UASs. Symptoms compatible with bronchiectasis (productive cough, but also fatigue, exercise intolerance and dyspnoea) are difficult to differentiate from those of CRS. Therefore, determination of the order of onset of the pathologies is difficult, although it is more reasonable that CRS preceded the development of bronchiectasis.

The finding of similar lung function among patients with bronchiectasis and CRS contrasts with previous reports. In a Chinese cohort with a low (35\%) prevalence of CRS among patients with bronchiectasis, patients with CRS had lower FEV1 and more prevalent colonisation with P. aeruginosa [7]. Conversely, and similar to our findings, GUILEMANy et al. [11] found a high prevalence of CRS, yet did not find differences in either lung function or $P$. aeruginosa colonisation among patients with bronchiectasis, with or without CRS. Regional differences in P. aeruginosa exposure may possibly explain the differences in the findings.

In the current study, patients with UASs had a significantly higher eosinophil count in peripheral blood and a higher (not statistically significant) total IgE. These differences, which are compatible with an allergic tendency, were more pronounced in the analyses that were based on positive CT findings. Allergic features are well known in CRS $[13,17]$ and evidence of eosinophil activation (elevated eosinophil cationic protein) was found among 14 patients with bronchiectasis [27]. However, most evidence points to a neutrophilic, rather than eosinophilic, inflammation in bronchiectasis [28, 29]. This study did not allow determining whether allergic inflammation is involved in the pathogenesis of bronchiectasis, as well as CRS, in patients with both features.

Several limitations to our study are worth mentioning. First, due to the retrospective design, we were not able to assess UASs systematically, but relied on previous clinical findings. Furthermore, sinus CT scans were not available for review for a large proportion of our patients. Therefore, the true involvement of the upper airway among patients with bronchiectasis may have been under- or overestimated. However, to the best of our knowledge, this is the first study of bronchiectasis that excludes patients with PCD and thus focuses on mechanisms other than ciliary dysfunction. This is also the first study to assess age of onset, eosinophilia and immunoglobulins, demonstrating earlier age of onset and an allergic tendency of patients with CRS and bronchiectasis.

Our hypothesis for the association of upper airway involvement with bronchiectasis is that CRS associated with allergy leads to the development of bronchiectasis. The mechanism may be either: 1) migration of secretions from the upper airway to the lower airway resulting in abnormal mucus clearance from the lower airway, 2) bacterial inflammation from the upper airway infecting the lower airway or 3) an inflammatory response originating in the upper airway and expanding to involve the lower airway. Each of these processes could be "entry points" into the "vicious cycle" hypothesis of bronchiectasis [30]. Of course, more than one of these mechanisms may be responsible. More studies are needed to test whether this hypothesis is true.

In conclusion, the involvement of the upper airway in patients with bronchiectasis is associated with an early age of onset and allergic features. Despite similar sputum bacteriology and lung function, patients with upper airway involvement had significantly more frequent exacerbations. More studies are needed to 
determine whether CRS is the cause of bronchiectasis or whether a common (allergic) mechanism predisposes to both diseases.

\section{Acknowledgements}

Author contributions: Study design and conduction: M. Shteinberg, J. Jrbashyan, N. Uri and Y. Adir. Ear, nose and throat assessment: J. Jrbashyan. Radiological scoring of CT scans: N. Nassrallah. Statistical analysis: N. Stein. All authors reviewed and authorised the manuscript.

\section{References}

1 Bourdin A, Gras D, Vachier I, et al. Upper airway 1: allergic rhinitis and asthma: united disease through epithelial cells. Thorax 2009; 64: 999-1004.

2 Licari A, Caimmi S, Bosa L, et al. Rhinosinusitis and asthma: a very long engagement. Int J Immunopathol Pharmacol 2014; 27: 499-508.

3 Jarvis D, Newson R, Lotvall J, et al. Asthma in adults and its association with chronic rhinosinusitis: the GA ${ }^{2}$ LEN survey in Europe. Allergy 2012; 67: 91-98.

4 Shapiro AJ, Zariwala MA, Ferkol T, et al. Diagnosis, monitoring, and treatment of primary ciliary dyskinesia: PCD foundation consensus recommendations based on state of the art review. Pediatr Pulmonol 2016; 51: 115-132.

5 Guilemany JM, Mariño-Sánchez FS, Angrill J, et al. The importance of smell in patients with bronchiectasis. Respir Med 2011; 105: 44-49.

6 Ramakrishnan VR, Ferril GR, Suh JD, et al. Upper and lower airways associations in patients with chronic rhinosinusitis and bronchiectasis. Int Forum Allergy Rhinol 2013; 3: 921-927.

7 Guan W, Gao Y, Li H, et al. Impacts of co-existing chronic rhinosinusitis on disease severity and risks of exacerbations in Chinese adults with bronchiectasis. PLoS One 2015; 10: e0137348.

8 Guilemany JM, Angrill J, Alobid I, et al. United airways: the impact of chronic rhinosinusitis and nasal polyps in bronchiectasic patient's quality of life. Allergy 2009; 64: 1524-1529.

9 Shirahata Y. [Correlation between upper airway tract and lower airway tract in the break down of sinobronchiectasis.] Nihon Jibiinkoka Gakkai Kaiho 1990; 93: 1991-1998.

10 King PT, Holdsworth SR, Freezer NJ, et al. Characterisation of the onset and presenting clinical features of adult bronchiectasis. Respir Med 2006; 100: 2183-2189.

11 Guilemany JM, Alobid I, Angrill J, et al. The impact of bronchiectasis associated to sinonasal disease on quality of life. Respir Med 2006; 100: 1997-2003.

12 Shoemark A, Ozerovitch L, Wilson R. Aetiology in adult patients with bronchiectasis. Respir Med 2007; 101: $1163-1170$.

13 Benninger M, Ferguson B, Hadley J, et al. Adult chronic rhinosinusitis: definitions, diagnosis, epidemiology, and pathophysiology. Otolaryngol Head Neck Surg 2003; 129: S1-S32.

14 De Boeck K, Wilschanski M, Castellani C, et al. Cystic fibrosis: terminology and diagnostic algorithms. Thorax 2006; 61: 627-635.

15 Reiff DB, Wells AU, Carr DH, et al. CT findings in bronchiectasis: limited value in distinguishing between idiopathic and specific types. Am J Roentgenol 1995; 165: 261-267.

16 Lund V, Kennedy D. Staging for rhinosinusitis. Otolaryngol Head Neck Surg 1997; 117: S35-S40.

17 Fokkens WJ, Lund VJ, Mullol J, et al. EPOS 2012: European position paper on rhinosinusitis and nasal polyps 2012. A summary for otorhinolaryngologists. Rhinology 2012; 50: 1-12.

18 Hopkins C, Browne JP, Slack R, et al. The Lund-Mackay staging system for chronic rhinosinusitis: how is it used and what does it predict? Otolaryngol Head Neck Surg 2007; 137: 555-561.

19 Lin S-H, Kuo P-H, Hsueh P-R, et al. Sputum bacteriology in hospitalized patients with acute exacerbation of chronic obstructive pulmonary disease in Taiwan with an emphasis on Klebsiella pneumoniae and Pseudomonas aeruginosa. Respirology 2007; 12: 81-87.

20 Pasteur MC, Bilton D, Hill AT, et al. British Thoracic Society guideline for non-CF bronchiectasis. Thorax 2010; 65: Suppl. 1, i1-i58.

21 Shashy RG, Moore EJ, Weaver A. Prevalence of the chronic sinusitis diagnosis in Olmsted County, Minnesota. Arch Otolaryngol Head Neck Surg 2004; 130: 320-323.

22 Pilan RRM, Pinna FR, Bezerra TFP, et al. Prevalence of chronic rhinosinusitis in Sao Paulo. Rhinology 2012; 50: $129-138$.

23 Nicotra MB, Rivera M, Dale AM, et al. Clinical, pathophysiologic, and microbiologic characterization of bronchiectasis in an aging cohort. Chest 1995; 108: 955-961.

24 Pasteur MC, Helliwell SM, Houghton SJ, et al. An investigation into causative factors in patients with bronchiectasis. Am J Respir Crit Care Med 2000; 162: 1277-1284.

25 Metersky ML. The initial evaluation of adults with bronchiectasis. Clin Chest Med 2012; 33: 219-231.

26 Campbell R. Managing upper respiratory tract complications of primary ciliary dyskinesia in children. Curr Opin Allergy Clin Immunol 2012; 12: 32-38.

27 Kroegel C, Schüler M, Förster M, et al. Evidence for eosinophil activation in bronchiectasis unrelated to cystic fibrosis and bronchopulmonary aspergillosis: discrepancy between blood eosinophil counts and serum eosinophil cationic protein levels. Thorax 1998; 53: 498-500.

28 Chalmers JD, Hill AT. Mechanisms of immune dysfunction and bacterial persistence in non-cystic fibrosis bronchiectasis. Mol Immunol 2013; 55: 27-34.

29 Schaaf B, Wieghorst A, Aries SP, et al. Neutrophil inflammation and activation in bronchiectasis: comparison with pneumonia and idiopathic pulmonary fibrosis. Respiration 2000; 67: 52-59.

30 Cole PJ. Inflammation: a two-edged sword - the model of bronchiectasis. Eur J Respir Dis Suppl 1986; 147: 6-15. 